\title{
Kaptive 2.0: updated capsule and LPS locus typing for the Klebsiella pneumoniae species complex
}

\author{
Margaret M. C. $\operatorname{Lam}^{1 *}\left(\right.$ 0000-0001-9590-6849), Ryan R. Wick ${ }^{1(0000-0001-8349-0778)}$, \\ Louise M. Judd ${ }^{1 \text { (0000-0003-3613-4839) }}$, Kathryn E. Holt ${ }^{1,2(0000-0003-3949-2471)}$ and \\ Kelly L. Wyres ${ }^{1 *}$ (0000-0002-4033-6639)
}

${ }^{1}$ Department of Infectious Diseases, Central Clinical School, Monash University, Melbourne, Victoria, 3004, Australia

${ }^{2}$ Department of Infection Biology, Faculty of Infectious and Tropical Diseases, London School of Hygiene \& Tropical Medicine, London WC1E 7HT, UK

Corresponding authors: Margaret.lam@monash.edu; Kelly.wyres@monash.edu

Keywords: Klebsiella pneumoniae, Klebsiella capsule K-locus genomic surveillance, K-locus, O-locus

\section{Repositories:}

\subsection{Repositories:}

Genome sequence from which the novel K locus KL182 was defined has been deposited under the accession JAJHNT000000000.

\begin{abstract}
The outer polysaccharide capsule and lipopolysaccharide antigens are key targets for novel control strategies targeting Klebsiella pneumoniae and related taxa from the $K$. pneumoniae species complex (KpSC), including vaccines, phage and monoclonal antibody therapies. Given the importance and growing interest in these highly diverse surface antigens, we had previously developed Kaptive, a tool for rapidly identifying and typing capsule $(\mathrm{K})$ and outer lipopolysaccharide $(\mathrm{O})$ loci from whole genome sequence data. Here, we report two significant updates, now freely available in Kaptive 2.0 (github.com/katholt/kaptive); i) the addition of 16 novel $\mathrm{K}$ locus sequences to the $\mathrm{K}$ locus reference database following an extensive search of $>17,000 \mathrm{KpSC}$ genomes; and ii) enhanced $\mathrm{O}$ locus typing to enable prediction of the clinically relevant $\mathrm{O} 2$ antigen (sub)types, for which the genetic determinants have been recently described. We applied Kaptive 2.0 to a curated dataset of $>12,000$ public KpSC genomes to explore for the first time the distribution of predicted $O$ (sub)types across species,
\end{abstract}


sampling niches and clones, which highlighted key differences in the distributions that warrant further investigation. As the uptake of genomic surveillance approaches continues to expand globally, the application of Kaptive 2.0 will generate novel insights essential for the design of effective KpSC control strategies.

\section{Significance as a BioResource to the community}

Klebsiella pneumoniae is a major cause of bacterial healthcare associated infections globally, with increasing rates of antimicrobial resistance, including strains with resistance to the drugs of last resort. The latter have therefore been flagged as priority pathogens for the development of novel control strategies.

K. pneumoniae produce two key surface antigen sugars (capsular polysaccharide and lipopolysaccharide (LPS)) that are immunogenic and targets for novel controls such as a vaccines and phage therapy. However, there is substantial antigenic diversity in the population and relatively little is understood about the distribution of antigen types geographically and among strains causing different types of infections. Whereas laboratory-based antigen typing is difficult and rarely performed, information about the relevant synthesis loci can be readily extracted from whole genome sequence data. We have previously developed Kaptive, a freely available tool for rapid typing of Klebsiella capsule and LPS loci from genome sequences.

Kaptive is now used widely in the global research community and has facilitated new insights into Klebsiella capsule and LPS diversity. Here we present an update to Kaptive facilitating i) the identification of 16 additional novel capsule loci, and ii) the prediction of immunologically relevant LPS $\mathrm{O} 2$ antigen subtypes. These updates will enable enhanced sero-epidemiological surveillance for K. pneumoniae, to inform the design of vaccines and other novel Klebsiella control strategies.

\section{Data summary}

1. The updated code and reference databases for Kaptive are available at https://github.com/katholt/Kaptive

2. Genome accessions from which reference sequences of novel $\mathrm{K}$ loci were defined are listed in Supplementary Table 1, and genomes from which these loci were detected (along with the corresponding Kaptive output) are listed in Supplementary Table 2.

3. Accessions for the genomes screened for $O$ types/subtypes (along with the corresponding Kaptive output) are listed in Supplementary Table 3.

The authors confirm all supporting data, code and protocols have been provided within the article or through supplementary data files. 


\section{Introduction}

70 The Klebsiella pneumoniae species complex (KpSC) is a group of closely related Gram-negative

bacterial taxa including the opportunistic pathogen, Klebsiella pneumoniae [1]. The ' $\mathrm{K}$ ' in the ESKAPE pathogens, $K$. pneumoniae is considered one of the six most important causes of drug resistant healthcare-associated infections [2], and antimicrobial resistant strains contribute significantly to the total burden of communicable disease in high income countries [3]. In low- and middle-income countries $K$. pne umoniae is also recognised as the leading cause of Gram-negative neonatal sepsis, contributing to $10 \%$ of total neonatal sepsis deaths $[4,5]$. K. pneumoniae with resistance to the third-generation cephalosporins and carbapenems are disseminating globally and of particular concern because they cause infections with very limited treatment options. As a consequence, there is increasing interest in developing novel anti-KpSC control strategies such as vaccines, phage and monoclonal antibody therapies [6-9].

The KpSC polysaccharide capsule and lipopolysaccharide (LPS) antigens are pathogenicity factors [10-13] and among the key targets for novel control strategies [7, 14-18]. Of particular interest, there is mounting evidence that capsule and/or LPS immunisation can elicit a protective immune response, and several anti-KpSC capsule and/or LPS vaccines have entered clinical trials [19-23]. However, considerable antigenic diversity exists within the KpSC population ( $>77$ serologically defined capsule types [24-26], >8 LPS 0 antigen serotypes [16, 27], and many more predicted on the basis of genomic data $[28,29])$, and there is a paucity of sero-epidemiological knowledge, which hampers efficient vaccine design. While traditional KpSC serological typing techniques are technically challenging and rarely performed, information about capsule and $\mathrm{O}$ antigen serotypes can be rapidly extracted from whole genome sequence (WGS) data by typing the corresponding capsule (K) and $O$ biosynthesis loci $[29,30]$.

The $\mathrm{K}$ locus comprises a $\sim 10-30 \mathrm{kbp}$ region of the chromosome and $\sim 10-30$ genes. The conserved capsule synthesis and export machinery genes are located in the $5^{\prime}$ - (galF, cpsACP, wzi, wza, wzb, $w z c$ ) and $3^{\prime}-($ ugd) most regions, and flank the genes encoding the capsule-sugar-specific synthesis machinery, Wzx capsule-specific flippase, and Wzy repeat unit polymerase [31, 32]. The $O$ locus comprises a $\sim 7-13 \mathrm{kbp}$ region of the chromosome between the $\mathrm{K}$ locus and the hisE gene [28]. All O loci identified to-date contain the wzm and wzt genes encoding the membrane transporter complex [33], but the relative positions of these genes is not consistent [28]. 
In light of these findings, we previously leveraged a collection of $>2500 \mathrm{KpSC}$ genomes to identify additional $\mathrm{K}$ loci, defined on the basis of unique gene content [28, 29]. A total of 59 loci were identified, and labelled KL101-KL159. While the corresponding serotypes remain uncharacterised, we predict that these loci encode distinct capsule types that likely correspond to the majority of the $10-70 \%$ of strains that have been deemed non-typeable and/or cross-reactive via serological typing techniques [34-36].

To facilitate the rapid identification of $\mathrm{K}$ loci from KpSC whole genome sequences, we developed a tool known as Kaptive [29], which uses a combination of BLASTn and tBLASTn search to identify the best matching locus from the reference database, and provide an indication of the match confidence. We recommend reporting matches of 'Good' confidence or higher. Low or no confidence matches generally result from sequencing and/or assembly problems that cause the $K$ locus to be split across multiple assembled contigs, but can also represent novel loci that were not captured in the original discovery genome set. Indeed, subsequent studies have identified 11 additional loci that have been incorporated into the database ([37] and see details at github.com/katholt/kaptive), resulting in a total of 146 loci defined to-date. We anticipate the discovery of additional novel loci, particularly given the availability of greater numbers of KpSC genomes from diverse sources and locations. Notably, our recent analysis of $>13,000$ publicly available KpSC genomes identified $19 \%$ with low or no confidence $\mathrm{K}$ locus matches that remain to be explored [38].

The majority of distinct LPS $\mathrm{O}$ antigens are also associated with distinct $\mathrm{O}$ loci (also known as rfb loci), distinguished on the basis of gene content [39-42]. However, key exceptions are strains expressing the $\mathrm{O} 1$ and $\mathrm{O} 2$ antigens, both of which can be associated with either of two distinct $\mathrm{O}$ loci, 01/O2v1 and 01/02v2 [15, 41,43]. Expression of either locus results in the production of an 02 antigen (D-galactan I or III), that can be converted to 01 by addition of a D-galactan II repeat unit by WbbYZ (encoded elsewhere in the genome) [44].

In 2018 we extended Kaptive to support KpSC O locus typing and included discrimination of the 01 and $\mathrm{O} 2$ types by tBLASTn search for the wbbYZ genes, reported as shown in Table 1 [30]. However, recent experimental evidence has shown that only the WbbY protein is needed to convert $\mathrm{O} 2$ to 01 [45]. Additionally, the genetic determinants of four distinct 02 antigen subtypes have now been fully elucidated (O2a, O2afg, $\mathrm{O} 2 \mathrm{ac}, \mathrm{O} 2 \mathrm{aeh})[45,46]$. The $\mathrm{O} 2 \mathrm{a}$ antigen comprises alternating repeat units of $\alpha$-(1-3)-linked galactopyranose (Galp) and $\beta$-(1-3)-linked galactofuranose (Galf) residues (Dgalactan I) [40]. O2afg comprises O2a with a (1-4)-linked Galp side chain, while O2aeh comprises O2a with a (1-2)-linked Galp side chain [43]. Like 01, O2ac comprises 02a or O2afg with an additional repeat unit covalently linked to the non-reducing terminus (it also seems likely that O2aeh can be modified in this way). While the 01 repeat unit comprises [-3)- $\alpha$-D-Galp-(1-3)- $\beta-D-G a l p-(1-]$ (also 
143 known as D-galactan II) [40], the O2ac repeat unit comprises [-3)- $\beta-D-G l c p N A c-(1-5)-\beta-D-G a l f(1-]$

$144[43,46]$. These subtypes are associated with specific combinations of 0 loci (01/02v1, 01/O2v2 and

145 a novel locus identified in the O2aeh type strain, here called 01/02v3) with/without additional

146 genes located elsewhere in the genome ( $w b b Y, g m I A B D$ and $w b m V W[45,46]$, see Table 1). There is

147 emerging evidence that these subtypes differ in terms of immunogenicity $[15,17,47]$ but little is

148 known about their distribution in the KpSC population, which may have implications for the design

149 of vaccines or monoclonal antibody therapies targeting KpSC LPS.

Here we report; i) an update to the KpSC K locus database to include 16 novel loci identified from a

high-throughput screen of $>17,000$ publicly available genomes; and ii) an updated version of Kaptive

investigations. We apply this update to explore the distribution of $O$ (sub)types between KpSC

species and among isolates from different sources and clonal groups (CGs), including the well-known

globally distributed multi-drug resistant clones and the hypervirulent clones associated with severe

community-acquired disease.

158

Table 1: Genetic determinants of Klebsiella pneumoniae species complex 01 and 02 outer

\begin{tabular}{|c|c|c|c|c|}
\hline \multirow{2}{*}{0 locus } & \multirow{2}{*}{ Extra genes } & \multirow{2}{*}{$\begin{array}{c}\text { Kaptive }<\text { v2.0 } \\
\text { Locus }^{\mathrm{a}}\end{array}$} & \multicolumn{2}{|c|}{ Kaptive $\geq v 2.0$} \\
\hline & & & Locus $^{a}$ & Type $^{\text {b }}$ \\
\hline 01/02v1 & none & O2v1 & 01/02v1 & O2a \\
\hline 01/02v2 & none & O2v2 & $01 / 02 v 2$ & O2afg \\
\hline 01/02v3 & none & N/A & $01 / 02 v 3$ & $02 a$ \\
\hline 01/02v1 & $w b b Y Z^{c}$ & 01v1 & 01/02v1 & 01 \\
\hline $01 / 02 v 2$ & $w b b Y Z^{c}$ & $01 v 2$ & $01 / 02 v 2$ & 01 \\
\hline $01 / 02 v 3$ & $w b b Y Z^{c}$ & N/A & $01 / 02 v 3$ & 01 \\
\hline 01/02v1 & $w b b Y$ OR $w b b Z$ & 01/02v1 & N/A & N/A \\
\hline $01 / 02 v 2$ & $w b b Y$ OR $w b b Z$ & $01 / 02 v 2$ & N/A & N/A \\
\hline
\end{tabular}




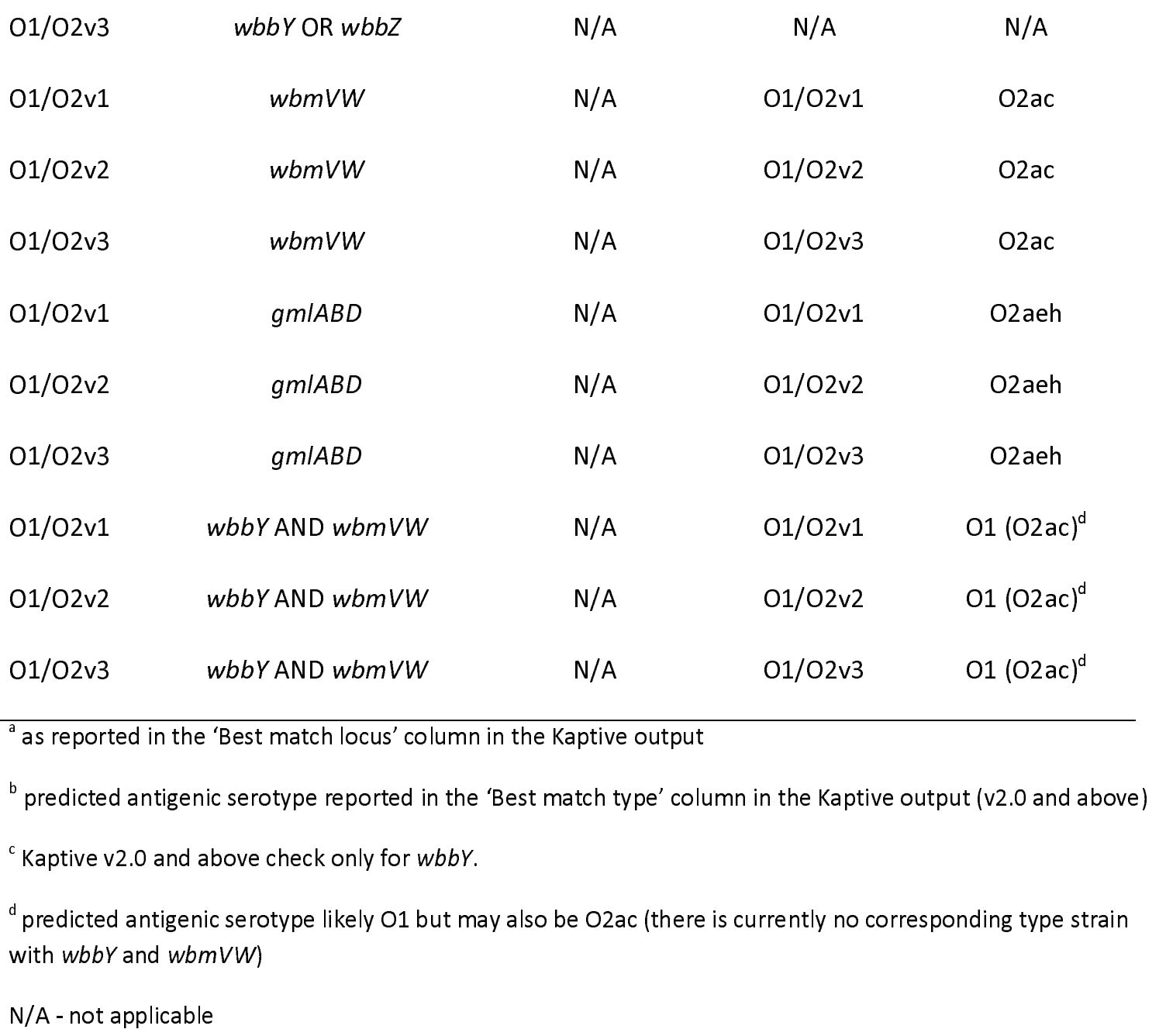

\section{Methods}

\section{$170 \quad$ Identification of novel $\mathrm{K}$ loci}

171 We have previously reported genotyping information for 13,156 publicly available KpSC genomes 172 (including species, multi-locus sequence types (STs), K loci and K locus confidence calls) [38]. Here 173 we leverage these data in combination with 3,958 additional published KpSC genomes and 638 from 174 our private collection (Table 2) for which the corresponding sequence reads were de novo 175 assembled using Unicycler v0.4.7. Species and STs were determined using Kleborate v2.0.3 [38] and 176 K loci identified using Kaptive v0.7.3 [29]. 
Collection description

Curated, publicly available KpSC isolate genomes

$\mathrm{KpSC}$ isolate genomes from diverse niches in a single city

in Italy

Blood and urinary tract KpSC isolate genomes from

Norwegian hospitals

Clinical KpSC isolate genomes from an Australian hospital

Community rectal carriage KpSC isolate genomes from

Norway

KpSC neonatal sepsis isolate genomes from south east

Asia and Africa

KpSC isolate genomes from pigs and pig farmers in

Thailand

Klebsiella variicola from various sources

\section{Reference}

[38] (also see

refs therein)

[48]

[49]

This study

[50]

[5]

[51]

[52]
N Genomes (N low confidence $\mathrm{K}$ loci $^{\mathrm{a}}$ )

$13156(1802)$

$1965(75)$

$868(358)$

$638(18)$

$484(36)$

$276(93)$

$253(31)$

$112(6)$

Total

17752 (2419)

181

${ }^{a}$ Genomes harbouring K loci for which the Kaptive confidence call was 'Low' or 'None', and subsequently included in this study.

Genomes for which the Kaptive K locus confidence call was "Low" or "None" were included for further analysis as follows: genomes for which the Kaptive output did not indicate a fragmented $\mathrm{K}$ locus assembly (i.e. the K locus problems column did not contain '?') were subjected to manual inspection using Bandage v0.8.1 [53] to visualise the BLASTn coverage to the best match $\mathrm{K}$ locus and assess if the genome truly harboured the best match locus, a variant thereof (insertion sequence (IS) or deletion variant) or a putative novel locus. Genomic regions corresponding to putative novel loci were extracted and clustered using CD-HIT-EST v4.8.1 (default parameters) [54]. A single representative of each cluster was; a) annotated using Prokka v1.14.6 [55] with a reference database of known KpSC K locus genes; b) subjected to BLASTn search for known KpSC K locus genes and those annotated in each of the other putative novel $\mathrm{K}$ loci. Inspection of the BLASTn results highlighted putative novel loci with similarity to each other and/or existing loci (i.e. those with 
BLASTn hits $\geq 80 \%$ identity and $\geq 80 \%$ coverage to multiple capsule-sugar-specific synthesis genes from the same reference locus). The corresponding loci were subsequently compared by BLASTn and visualised with the Artemis Comparison Tool v18.0.2 [56] to clarify if they were distinct or should be considered as IS or deletion variants of the same locus.

Annotations were manually curated for one representative of each of the final set of distinct novel loci (Supplementary Table 1). Where possible, sequences without IS transposase annotations were preferentially selected for curation, as is recommended in order to prevent Kaptive reporting spurious gene matches to transposases that may be present in multiple copies in any location of a query genome. Where no IS transposase-free representative was available $(n=5$ loci, subsequently assigned KL173, KL176, KL181, KL184 and KL185), full length ISs were identified by BLASTn search of the ISfinder database [57] and manually deleted along with their associated direct repeats and one copy of the associated target site duplicated repeat to obtain a putative IS-free reference sequence. Curated locus annotations were added to the reference database and Kaptive was rerun on all genomes to determine the prevalence of novel loci (Supplementary Table 2). Only Kaptive locus calls with confidence 'Good' or better are reported. Visual comparisons of the novel K loci and annotated coding sequences (CDS) were generated with clinker v0.0.21 [58].

\section{Implementation of 02 subtyping}

Kaptive takes as input a query genome assembly and a reference locus database in GenBank format. Loci are identified by a note field within the sequence source information in the format:

/note="O locus: 01/02v1"

Additional genes relevant to KpSC O typing (i.e those located outside of the $\mathrm{O}$ locus) are marked as follows:

/note="Extra genes: wbbY"

In Kaptive 2.0 we have implemented an additional note field for reference loci that indicates the corresponding serotype. E.g. for the 05 locus the corresponding $O$ type is known to be 05 and hence the corresponding note fields read:

/note="O locus: O5" 
/note="O type: O5"

230 For loci identified from genome data and defined on the basis of gene content alone, e.g. OL101,

231 there is no known serotype and hence the corresponding note fields read:

/note="O locus: OL101"

/note="O type: unknown (OL101)"

For the $\mathrm{O} 1$ and $\mathrm{O} 2$ loci, the corresponding serotypes/subtypes are distinguished by the presence/absence of additional genes located elsewhere in the genome, as is now indicated by the use of the term 'special logic' in the serotype notes field e.g:

/note="O locus: 01/02v1"

/note="O type: special logic"

After identifying the best match locus from the database (the locus with the highest BLASTn coverage), Kaptive 2.0 will extract the corresponding type information from the GenBank annotation. When the type is indicated as 'special logic' Kaptive will perform tBLASTn search for all coding sequences within database entries marked with the note field 'Extra genes' (wbbY, Genbank accession MG458672.1; wbmVW, accession MG602074.1; gmIABD, accession MG458670.1). tBLASTn hits exceeding the minimum identity and coverage cut-offs (default $80 \%$ and $90 \%$, respectively) are interpreted to indicate the presence of the corresponding gene(s). The reported $O$ type is determined by the combination of best match $O$ locus and any extra gene(s) detected, as specified in the "Klebsiella_o_locus_primary_reference.logic" file and shown in Table 1. In the event that a combination is not represented in the logic file, Kaptive will report the $O$ type as "unknown."

\section{Updated locus and serotype reporting}

In the previous version of Kaptive the distinction between the KpSC 01 and $\mathrm{O} 2$ types was indicated in the output table in the 'Best match locus' as shown in Table 1. However, we have noted that this has resulted in confusion in the community regarding the distinction between $O$ loci and $O$ types. In order to clarify these differences and ensure that all relevant information is included in the output, output (see Table 1). The three distinct $\mathrm{O}$ loci associated with the $\mathrm{O} 1$ and $\mathrm{O} 2$ (sub)types are reported simply as 01/02v1, 01/02v2 and 01/02v3 in the 'Best match locus' column, meaning that genomes harbouring the same $O$ locus can be easily identified even when predicted to express a different $O$ 
The same approach for distinguishing 'Best match locus' from 'Best match type' is applied to all databases parsed by Kaptive 2.0 in order to aid interpretation of the output and clarify what is known about the relationships between loci and serotypes. In particular we hope this will clarify where serotypes are or are not known. To enable backwards compatibility for reference locus database that do not contain serotype note fields, the corresponding types are left blank if no type information is available.

\section{Exploring the distribution of $O$ (sub)types}

We used Kaptive 2.0 to identify $\mathrm{O}$ loci and $\mathrm{O}$ types for 10,734 non-redundant KpSC genomes in the curated collection reported previously [38]. Additionally, we supplemented this collection with two systematically collected datasets of KpSC from underrepresented sources: 484 human gut carriage isolate genomes reported by Raffelsburger et al [50], and 875 non-human associated isolate genomes reported by Thorpe et al [48]. (We excluded human-associated KpSC genomes reported as part of the latter study because these data were biased by a significant overrepresentation of the ST307 and ST512 clones that were circulating in the local hospital and were already represented in high numbers in our curated genome collection.) Only Kaptive calls with confidence 'Good' or better are reported.

\section{Results and Discussion}

\section{Identification and characterisation of 16 novel $\mathrm{K}$ loci}

283 A total of 2,419 of $17,752 \mathrm{KpSC}$ genomes (13.6\%) had low or no confidence Kaptive $\mathrm{K}$ locus calls

284 (Figure 1), of which the vast majority $(2,129 ; 88 \%)$ were indicated as fragmented and 37 were 285 excluded because the $\mathrm{K}$ loci were likely to harbour an overrepresentation of homopolymer repeat 286 errors due to the assembly approach comprising Oxford Nanopore Technologies read data only. 287 Following manual inspection, 148 of the 253 remaining genomes were considered to match to 288 known K loci and 45 were unresolvable because the $\mathrm{K}$ locus was fragmented across multiple 289 assembly contigs. The former had not been confidently typed by Kaptive for a variety of reasons 290 including IS insertions, small- or large-scale deletions and/or numerous frameshift mutations that 291 were interpreted as missing genes. After exclusion of these genomes, 60 putative novel loci 292 remained and were grouped into 22 sequence clusters at $90 \%$ nucleotide identity. Sixteen clusters 293 were determined to represent true distinct and novel $\mathrm{K}$ loci, while 6 were identified as additional 294 IS/deletion variants of other putative novel loci or existing loci (Figure 1, see Methods). 


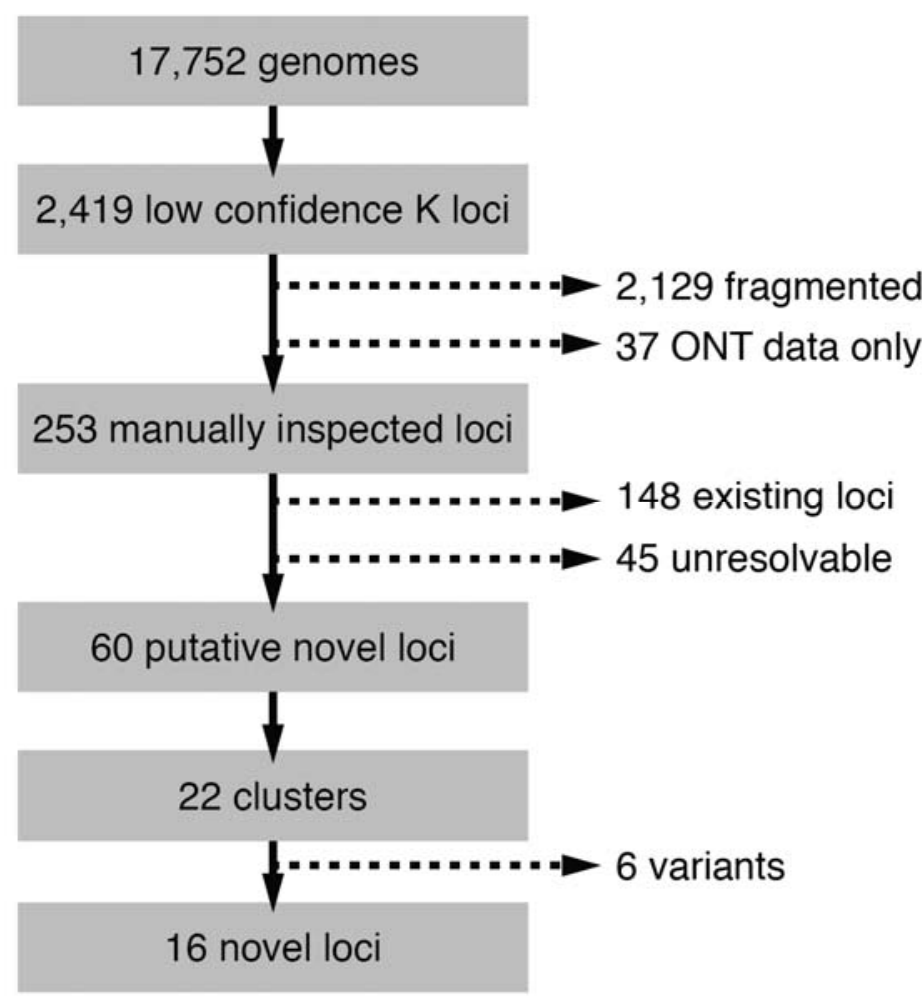

Figure 1: Process of identification of novel $K$ loci from 17,752 K. pneumoniae species complex genomes. Candidate genomes were identified as those with Kaptive K locus confidence calls 'Low' or 'None,' and were iteratively filtered to remove; i) fragmented or low quality locus sequences including 37 assembled using only Oxford Nanopore Technologies (ONT) data; ii) true matches to existing $\mathrm{K}$ loci; iii) insertion sequence and/or deletion variants of existing loci or other putative novel loci (see Methods for full details).

The final set of 16 novel loci were assigned KL171-KL186, and ranged in length from 22,624 to 30,215 bp and contained 19 to 25 genes (Figure 2). All loci contained the conserved capsule synthesis and export machinery genes; galF, $C P S A C P, w z a, w z b, w z c$, gnd and ugd; however, only 14 loci harboured wzi (present in $>98 \%$ of all previously reported KpSC K loci [29]). The Wzi protein is not considered essential for capsule production but plays a role in capsule surface attachment such that mutants lacking wzi produce lower levels of bound and higher levels of cell-free polysaccharide [59]. In the novel KL175 and KL176 loci, the wzi gene appeared to be replaced by four coding sequences predicted to encode two hypothetical proteins, a GfCC protein and a putative lipoprotein $\mathrm{YjbH}$ precursor. $\mathrm{N}=3 / 4$ of these sequences had $76-84 \%$ sequence identity to those present in the KL33 and KL40 reference loci, wherein wzi is also absent [32]. form a pore that allows the capsule to cross the cell membrane in a role similar to that of the KpSC 
316 Wza protein [60]. However, apparently intact copies of wza were also present in each of the KL33, $317 \mathrm{KL40}, \mathrm{KL175}$ and KL176 loci, so it is unclear what if any role GfCC plays in the export of these 318 capsules. Similarly, the role of the $\mathrm{YjbH}$ precursor protein is unclear. $\mathrm{YjbH}$ is indicated as a regulator 319 of exopolysaccharide expression in E. coli but it is encoded as part of the yjbEFGH [61] operon that 320 was absent from these KpSC K loci.

As expected, all novel loci harboured a variant of $w z x$ and of $w z y$, encoding the capsule flippase and repeat unit polymerase, respectively, as well as either wcaJ or wbaP (encoding the initiating glycosyl transferases) [31]. Ten loci (63\%) harboured the rmIBADC capsule-specific sugar synthesis genes responsible for the inclusion of dTDP-L-rhamnose in the capsule and $9(57 \%)$ harboured manBC associated with the inclusion of GDP-D-mannose [32]. The remaining capsule-specific sugar synthesis genes were each found in $\leq 5$ loci each.

We assessed the prevalence of the novel loci among the public genome collections (Table 2). Most ( $n=11 ; 69 \%$ ) were rare, identified in $\leq 5$ genomes. However, KL174, KL177, KL180, KL181 and KL183 were each detected in $\geq 7$ and up to 33 genomes. Notably, these loci were generally associated with multiple STs and geographies; n=14 KL174 genomes with 9 STs from 11 countries, n=8 KL177 genomes with 3 STs ( $n=6$ ST656) from 6 countries, $n=7$ KL180 genomes with 6 STs from 5 countries, $n=9$ KL181 genomes with 2 STs from 4 countries, and $n=33$ KL183 genomes with 22 STs from 14 countries. Further, 9 of the novel loci were detected in genomes sequenced from non-human samples including animal (KL172, KL173, KL175, KL176, KL181, KL183, KL186), environmental (KL178, KL181, KL183) and food sources (KL180, KL183). In particular, 89\% of KL181 genomes were sourced from non-human sources (migratory birds, bovine, swine, murine or soil).

While the vast majority of KpSC genomes can be confidently assigned $\mathrm{K}$ loci from the existing database, as greater numbers of KpSC genomes become available we anticipate that additional novel K loci will be identified. In particular, it is unlikely that the current database fully captures the diversity present among KpSC circulating in the environment and non-human hosts that remain comparatively underrepresented in current genome collections. Furthermore, Klebsiella $\mathrm{K}$ loci are thought to undergo frequent reassortment via homologous recombination and IS-mediated horizontal gene transfer both within and outside the Klebsiella genus [29, 62], providing a population surveillance. 
bioRxiv preprint doi: https://doi.org/10.1101/2021.11.05.467534; this version posted November 10, 2021. The copyright holder for this preprint

(which was not certified by peer review) is the author/funder, who has granted bioRxiv a license to display the preprint in perpetuity. It is made available under aCC-BY 4.0 International license.

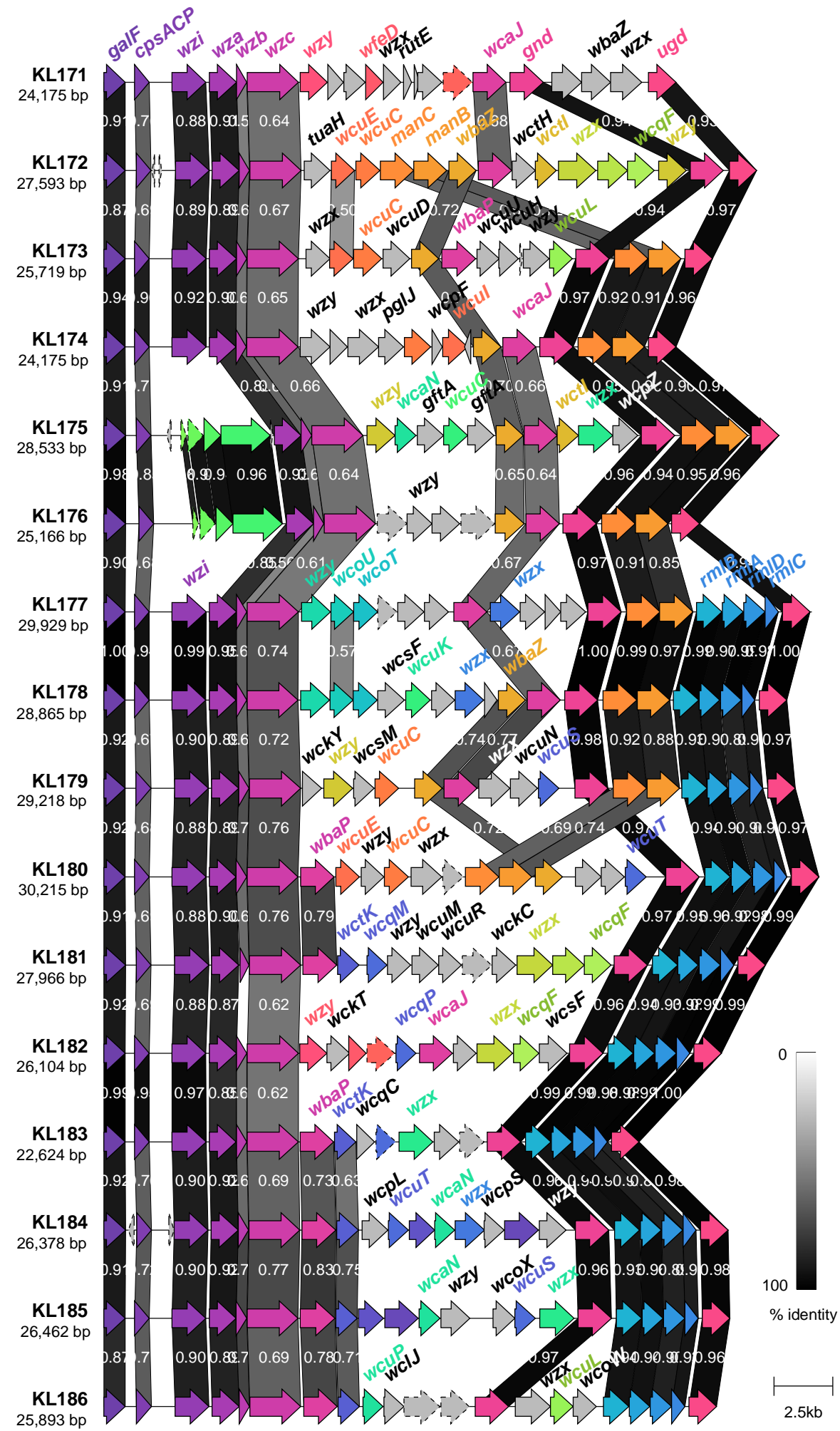


$350 \quad$ Figure 2: Genetic structure of novel $\mathrm{K}$ loci identified in this study. Coding sequences are represented by

351 arrows, labelled by their gene name where applicable, and coloured by homology (sequence identity $\geq 30 \%$ ).

352 Coding sequences predicted to encode hypothetical proteins are represented by arrows with a dashed outline.

353 Shading between the $\mathrm{K}$ loci represents regions of similarity between coding sequences as identified by clinker

354 [58], and the level of similarity indicated in the legend.

\section{$355 \quad$ o locus and 0 type distributions}

356 In 2018 we implemented KpSC O locus typing in Kaptive, with the capacity to distinguish the 01 and

$357 \quad 02$ antigen types [30]. In the latest version of Kaptive we have additionally implemented 02 antigen

358 subtype prediction (see Methods and Table 1). Subsequently, we used Kaptive to explore the

359 distribution of $O$ loci and predicted $O$ types among 12,093 publicly available KpSC genomes (see

360 Methods and Supplementary Table 3). A total of 11,571 genomes (95.7\%) were assigned O locus

361 calls with 'Good' confidence or better. Among these, the most common O loci were 01/02v2

$362(n=4,015,34.7 \%), 01 / 02 v 1(n=3,413,29.5 \%)$ and O3b ( $n=1,101,9.5 \%)$. The 01/02v3 locus, which

363 was added to the database as part of this work (first described in association with O2aeh, GenBank

364 accession MG280710.1 [46]), was identified in only 23 genomes (0.2\%).

366 The distribution of $\mathrm{O}$ loci differed by species (Figure 3 ), notably the $01 / 02$ loci were

367 overrepresented among K. pneumoniae compared to other species $\left(p<2.2 \times 10^{-16}\right.$, OR $64.3,95 \% \mathrm{Cl}$

368 48.9-86.1, by Fisher's Exact test), whereas the 03/03a and 05 loci were underrepresented among $K$.

369 pneumoniae $\left(p<2.2 \times 10^{-16}\right.$, OR 0.03, 95\% Cl 0.025-0.035; $p<2.2 \times 10^{-16}$, OR 0.05, 95\% Cl 0.042-0.060;

370 respectively by Fisher's Exact test). Within $K$. pneumoniae, $47.6 \%$ of the isolates carrying an $01 / 02$

371 locus were predicted to express an 01 antigen $(n=3,521 / 7,396)$, while $34.9 \%(n=2,581)$ and $16.6 \%$

$372(n=1,224)$ were predicted to express $02 \mathrm{afg}$ and $02 \mathrm{a}$, respectively. No isolates of any species were

373 predicted to express 02 aeh and only 75 isolates were predicted to express 02 ac (including $\mathrm{n}=69 \mathrm{~K}$.

374 pneumoniae). A single $K$. pneumoniae isolate carried the 01/02v1 locus in combination with the

$375 w b b Y$ gene (predicted to result in conversion of $02 a$ to 01 ), and the $w b m V W$ genes (predicted to

376 result in conversion of $\mathrm{O} 2 \mathrm{a}$ to $\mathrm{O} 2 \mathrm{ac}$ ), suggesting that this isolate (NCTC8849, from a respiratory tract

377 specimen collected in the United Kingdom in 1951) may be able to express both the 01 and the O2ac

378 antigens. However, there is evidence that expression of O2ac is thermoregulated, specifically

379 downregulated at $37^{\circ} \mathrm{C}$, hence it is likely that this strain expressed the 01 antigen within the human

380 host [46]. 
381

382

383

384

385

386

387 a

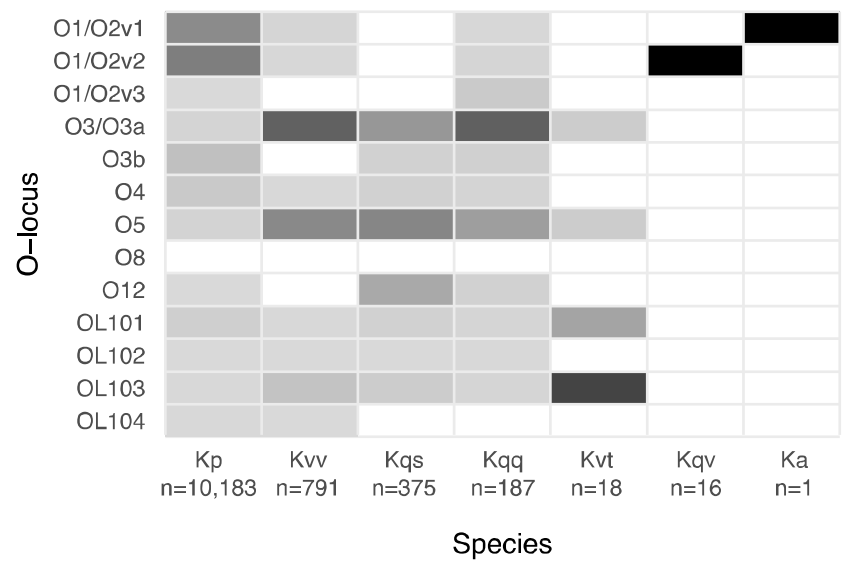

Prop genomes b

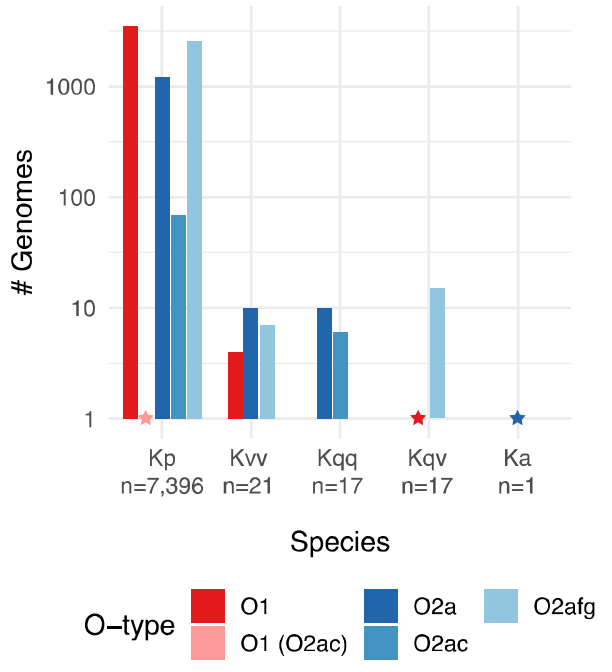

Figure 3: Distribution of $\mathrm{O}$ loci and predicted 01/O2 (sub)types by species. a) Heatmap showing the proportion of genomes of each species harbouring each distinct $O$ locus. Total sample sizes for each species are indicated below $\mathrm{x}$-axis labels. b) Bar graph showing the number of genomes of each species predicted to express $\mathrm{O} 1$ and $\mathrm{O} 2$ antigens. Only species for which at least one genome was predicted to express an $\mathrm{O} 1$ or $\mathrm{O} 2$ antigen are shown (total genomes indicated below $x$-axis labels). Stars indicate the position of bars of size 1. $\mathrm{Kp}, K$. pneumoniae; Kvv, K. variicola subsp. variicola; Kqs, $K$. quasipneumoniae subsp. similipneumoniae; Kqq, $K$. quasipneumoniae subsp. quasipneumoniae; Kvt, K. variicola subsp. tropica; Ka, K. africana.

Our data also revealed differences in the distribution of $O$ types by isolate source (Figure 4). Isolates from human specimens appeared to be enriched for types 01, 02a and/or O2afg compared to those from other hosts and/or environmental sources, which in part likely reflects the dominance of $K$. pneumoniae among clinical specimens. Interestingly, with the exception of liver abscess isolates, the prevalence of 02 a was similar across all human-associated source types $(8.5 \%-11.7 \%)$ whereas clinical isolates were generally enriched for O2afg compared to gut carriage isolates $(22.2 \%-30.1 \%$ vs. $14.2 \%, \mathrm{p}<2.2 \times 10^{-16}, \mathrm{OR} 0.49,95 \% \mathrm{Cl} 0.42-0.57$ using Fisher's Exact test of gut carriage isolates vs all clinical categories combined) and the prevalence of 01 appeared to be higher among invasive than non-invasive isolates ( $38.8 \%$ among blood/sterile site isolates, $32.0 \%$ among respiratory isolates, $28.2 \%$ among urinary tract isolates). Most strikingly, the overwhelming majority of liver abscess isolates (84.3\%) were predicted to express 01 . We speculated that the latter may be driven at least in part by the underlying population structure of $K$. pneumoniae causing liver abscess disease, which is dominated by a small number of so-called 'hypervirulent' clones. Indeed our current and previously reported data [28] showed that the most common of these clones (ST23, ST86, ST65 and ST25) were each associated with very high prevalence of $01(\geq 81 \%$, Figure 5$)$. The data also indicated that the majority of the well-known globally distributed MDR clones and other common clones were each associated with a single dominant $O$ (sub)type, however there was diversity between clones: ST14, ST15, ST20, ST29 and ST101 were each associated with 01 ( $\geq 77 \%$ 


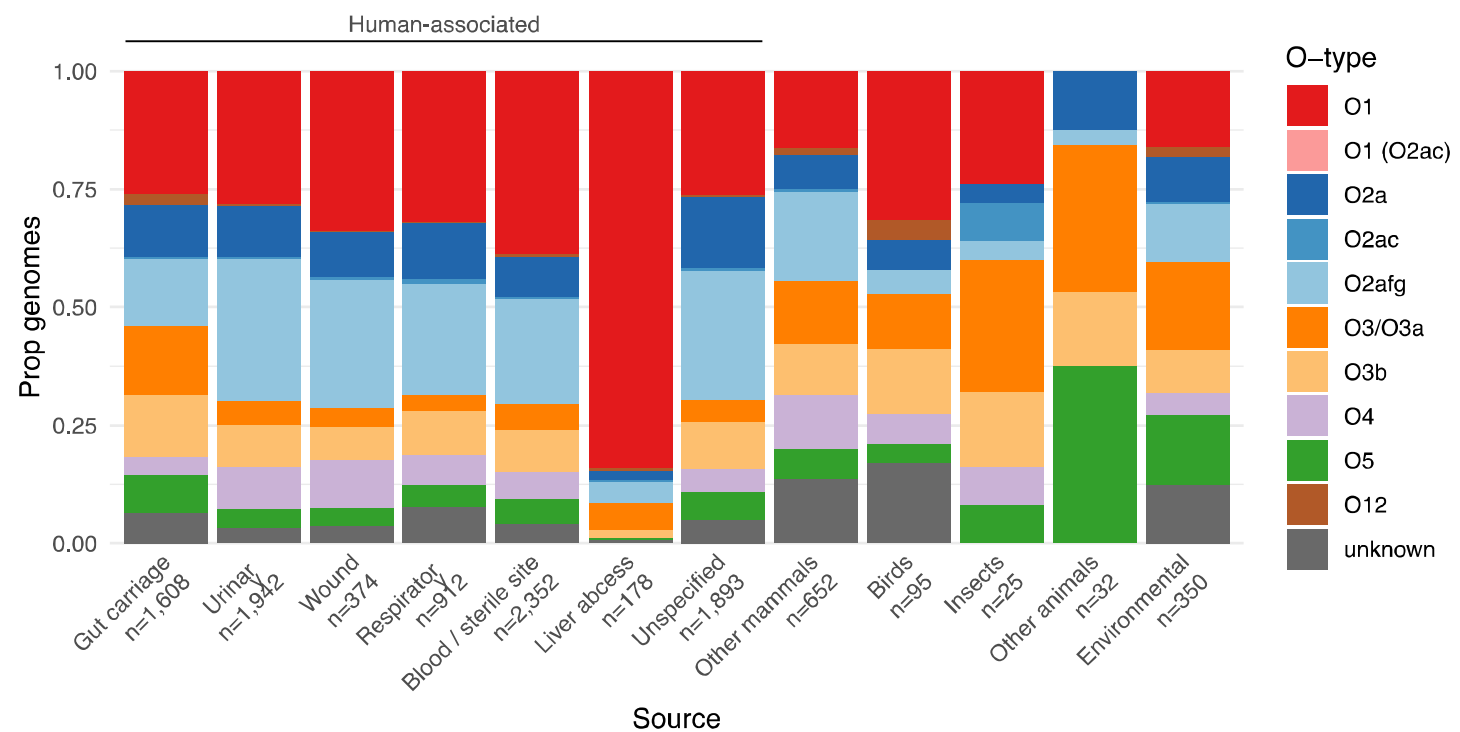

Source

Figure 4: Distribution of predicted $O$ types by isolate source. Bars show the proportion of isolates for each of 12 selected sources of interest that were predicted to encode each $O$ antigen (as indicated in the legend). The total number of isolates representing each source are indicated below the $\mathrm{x}$-axis. 


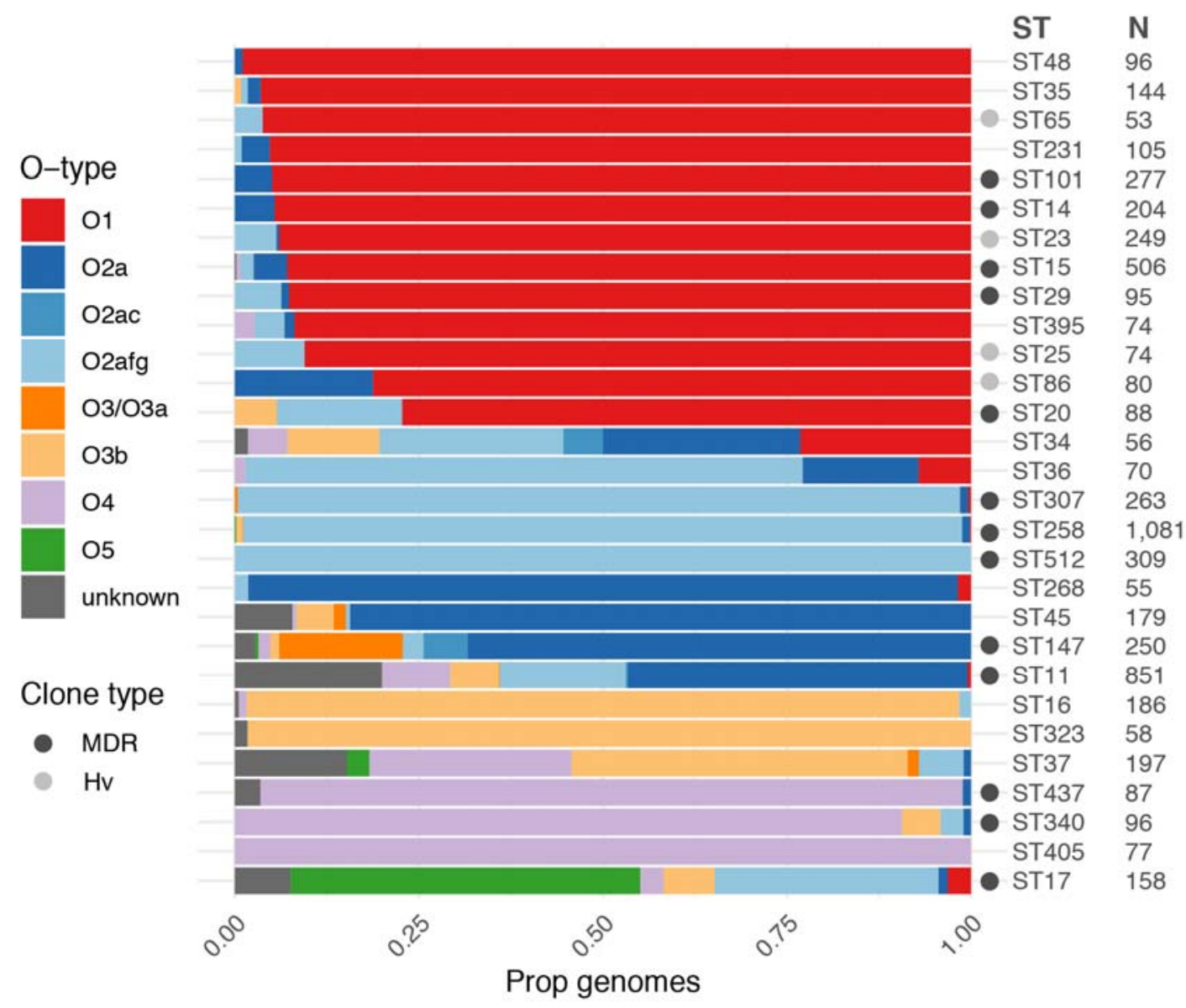

Figure 5: Distribution of predicted $O$ types among common $K$. pneumoniae clones. Bars show the proportion of genomes predicted to express each $O$ (sub)type within each clone (coloured as per legend). Sample sizes are indicated and only clones for which $\mathrm{N}>50$ are shown. Globally distributed multi-drug resistant (MDR) and hypervirulent (Hv) clones are indicated by grey circles as per legend (clones as described previously [1], additionally ST340 and ST437 belong to clonal group 258, ST16 belongs to clonal group 20)).

424 Previous reports have also indicated a dominance of 01 antigen among human clinical $K$. pneumoniae isolates $[27,28,63,64]$, followed by the 03 group in earlier reports $[27,63]$ and the 02 group in later reports $[28,64]$. While few prior studies have distinguished between 02 subtypes, a recent re-analysis of $573 \mathrm{KpSC}$ genome sequences reported in [28] indicated that $42 \%$ of the 387 genomes carrying an 01/02 O locus were predicted to express $02 \mathrm{afg}$, followed by 01 , a small number $02 \mathrm{a}$ or O2ac, and no O2aeh [46]. Additionally, the O2afg antigen has been previously associated with the ST258, ST512 and ST307 clones [15, 47]. Analysis of 276 neonatal sepsis $K$. pneumoniae genomes from South East Asia and Africa [5] revealed a dominance of 01 (54\%) followed by O2afg (14\%), 04 (11\%), O2a (7\%), and less than 5\% prevalence of 03/03a, O3b, 05 and 012. It has been suggested that the lower immunogenicity of O2afg compared to O2a and O1, may provide a selective advantage that has facilitated the widespread dissemination of these clones, i.e. 
435 by enabling immune evasion $[15,17]$; however, we note that our data show that several other highly

436 successful and widespread MDR clones are not associated with O2afg but instead are associated

437 with the highly immunogenic 01 (e.g. ST15, ST20, ST101), indicating that low 0 antigen

438 immunogenicity is not a requirement for widespread dissemination and/or other factors are also

439 playing a role (e.g. the interaction with the polysaccharide capsule).

\section{Conclusions}

We present updated KpSC K and O loci databases and an updated version of the Kaptive genotyping tool that allows rapid prediction of $\mathrm{O} 1$ and $\mathrm{O} 2$ antigen (sub)types from whole genome assemblies. Application of the updated approach to a collection of $>12,000$ publicly available KpSC genomes indicated key differences in the distribution of 01/02 antigens by isolate source and strong associations with the underlying $\mathrm{KpSC}$ population structure. Further investigation of these trends as well as the broader population sero-epidemiology is warranted to inform the effective design of novel KpSC control strategies. As genomic surveillance of KpSC continues to gain momentum,

449 Kaptive and its accompanying $\mathrm{K}$ and $\mathrm{O}$ locus databases are poised to play a key role in such 450 investigations.

\section{Author statements}

\section{Authors and contributors}

KEH and KLW were responsible for conceptualisation, supervision, project administration and acquisition of funding. RRW and KLW developed methodology, developed and/or tested software. LMJ performed DNA extractions and isolate sequencing. MMCL and KLW performed investigation, data curation and writing (original draft preparation). All authors performed writing (review and

\section{Conflicts of interest}

460 The authors declare that there are no conflicts of interest.

\section{Funding information}

This work was supported by the Bill and Melinda Gates Foundation (Investment Grant INV023041

464 awarded to KEH and KLW). KLW is supported by the National Health and Medical Research Council of 465 Australia (Investigator Grant APP1176192).

\section{References}

467 1. Wyres KL, Lam MMC, Holt KE. Population genomics of Klebsiella pneumoniae. Nat Rev 
Microbiol 2020;18:344-359.

Pendleton JN, Gorman SP, Gilmore BF. Clinical relevance of the ESKAPE pathogens. Expert Rev Anti Infect Ther 2013;11:297-308.

3. Cassini A, Högberg LD, Plachouras D, Quattrocchi A, Hoxha A, et al. Attributable deaths and disability-adjusted life-years caused by infections with antibiotic-resistant bacteria in the EU and the European Economic Area in 2015: a population-level modelling analysis. Lancet Infect Dis 2019;19:56-66.

4. Okomo U, Akpalu ENK, Le Doare K, Roca A, Cousens S, et al. Aetiology of invasive bacterial infection and antimicrobial resistance in neonates in sub-Saharan Africa: a systematic review and meta-analysis in line with the STROBE-NI reporting guidelines. Lancet Infect Dis 2019;19:1219-1234.

5. Sands K, Carvalho MJ, Portal E, Thomson K, Dyer C, et al. Characterization of antimicrobialresistant Gram-negative bacteria that cause neonatal sepsis in seven low- and middle-income countries. Nat Microbiol 2021;6:512-523.

6. World Health Organization. Global priority list of antibiotic-resistant bacteria to guide research, discovery, and devlopment of new antibiotics. 2017.

7. Motley MP, Fries BC. A new take on an old remedy: generating antibodies against bacteria in a postantibiotic world. $m S$ phere 2017;2:e00397-17.

8. Aleshkin A V., Ershova ON, Volozhantsev N V., Svetoch EA, Popova A V., et al. Phagebiotics in treatment and prophylaxis of healthcare-associated infections. Bacteriophage 2016;6:e1251379.

9. Assoni L, Girardello R, Converso TR, Darrieux M. Current stage in the development of 10. Cortés G, Borrell N, Astorza D, Gómez C, Sauleda J, et al. Molecular analysis of the contribution of the capsular polysaccharide and the lipopolysaccharide $O$ side chain to the virulence of Klebsiella pneumoniae in a murine model of pneumonia. Infect Immun 2002;70:2583-2590.

11. Lawlor MS, Hsu J, Rick PD, Miller VL. Identification of Klebsiella pne umoniae virulence determinants using an intranasal infection model. Mol Microbiol 2005;58:1054-1073.

12. Evrard B, Balestrino D, Dosgilbert A, Bouya-Gachancard JL, Charbonnel N, et al. Roles of capsule and lipopolysaccharide $O$ antigen in interactions of human monocyte-derived dendritic cells and Klebsiella pneumoniae. Infect Immun 2010;78:210-219.

13. March C, Cano V, Moranta D, Llobet E, Pérez-Gutiérrez C, et al. Role of bacterial surface structures on the interaction of Klebsiella pneumoniae with phagocytes. PLoS One 2013;8:116. 
14. D’Andrea MM, Marmo P, Henrici De Angelis L, Palmieri M, Ciacci N, et al. \$bO1E, a newly discovered lytic bacteriophage targeting carbapenemase-producing Klebsiella pneumoniae of the pandemic clonal group 258 clade II lineage. Sci Rep 2017;7:1-8.

15. Szijártó V, Guachalla LM, Hartl K, Varga C, Banerjee P, et al. Both clades of the epidemic KPC-producing Klebsiella pneumoniae clone ST258 share a modified galactan O-antigen type. Int J Med Microbiol 2016;306:89-98.

16. Guachalla LM, Stojkovic K, Hartl K, Kaszowska M, Kumar Y, et al. Discovery of monoclonal antibodies cross-reactive to novel subserotypes of $K$. pneumoniae 03. Sci Rep 2017;7:6635.

17. Pennini ME, De Marco A, Pelletier M, Bonnell J, Cvitkovic R, et al. Immune stealth-driven 02 serotype prevalence and potential for therapeutic antibodies against multidrug resistant Klebsiella pneumoniae. Nat Comms 2017;8:1991.

18. Seeberger PH, Pereira CL, Khan N, Xiao G, Diago-Navarro E, et al. A semi-synthetic glycoconjugate vaccine candidate for carbapenem-resistant Klebsiella pneumoniae. Angew Chem Int Ed Engl 2017;56:13973-13978.

19. Ravinder M, Liao KS, Cheng YY, Pawar S, Lin TL, et al. A synthetic carbohydrate-protein conjugate vaccine candidate against Klebsiella pneumoniae serotype K2. J Org Chem 2020;85:15964-15997.

20. Feldman MF, Mayer Bridwell AE, Scott NE, Vinogradov E, McKee SR, et al. A promising bioconjugate vaccine against hypervirulent Klebsiella pneumoniae. Proc Natl Acad Sci US A 2019;116:18655-18663.

21. Campbell WN, Hendrix E, Cryz S, Cross AS. Immunogenicity of a 24-valent Klebsiella capsular polysaccharide vaccine and an eight-valent Pseudomonas O-polysaccharide conjugate vaccine administered to victims of acute trauma. Clin Infect Dis 1996;23:179-181.

22. Hegerle N, Choi M, Sinclair J, Amin MN, Ollivault-Shiflett M, et al. Development of a broad spectrum glycoconjugate vaccine to prevent wound and disseminated infections with Klebsiella pneumoniae and Pseudomonas aeruginosa. PLoS One 2018;13:e0203143.

23. ClinicalTrials.gov [Internet]. Identifier: NCT04959344 Safety and Immunogenicity of a Klebsiella pneumoniae tetravalent bioconjugate vaccine (Kleb4V).

24. Edmunds PN. Further Klebsiella capsule types. J Infect Dis 1954;94:65-71.

25. Edwards PR, Fife MA. Capsule types of Klebsiella. J Infect Dis 1952;91:92-104.

26. Ørskov IDA, Fife-Asbury MA. New Klebsiella capsular antigen, K82, and the deletion of five of those previously assigned. Int J Syst Bacteriol 1977;27:386-387.

27. Trautmann M, Ruhnke M, Rukavina T, Held TK, Cross AS, et al. O-antigen seroepidemiology of Klebsiella clinical isolates and implications for immunoprophylaxis of Klebsiella infections. 
Clin Diag Lab Immun 1997;4:550-555.

28. Follador R, Heinz E, Wyres KL, Ellington MJ, Kowarik M, et al. The diversity of Klebsiella pneumoniae surface polysaccharides. Microb Genomics;2. Epub ahead of print 2016. DOI: 10.1099/mgen.0.000073.

29. Wyres KL, Wick RR, Gorrie C, Jenney A, Follador R, et al. Identification of Klebsiella capsule

31. Whitfield C. Biosynthesis and assembly of capsular polysaccharides in Escherichia coli. Annu Rev Biochem 2006;75:39-68.

32. Pan Y-J, Lin T-L, Chen C-T, Chen Y-Y, Hsieh P-F, et al. Genetic analysis of capsular synthesis loci from whole genome data. Microb Genomics;2. Epub ahead of print 2016. DOI: 10.1099/mgen.0.000102.

30. Wick RR, Heinz E, Holt KE, Wyres KL. Kaptive Web: user-friendly capsule and lipopolysaccharide serotype prediction for Klebsiella genomes. J Clin Microbiol 2018;56:e00197-18.

polysaccharide synthesis gene clusters in 79 capsular types of Klebsiella spp. Nat Sci Rep 2015;5:15573.

33. Cuthbertson L, Kimber MS, Whitfield C. Substrate binding by a bacterial ABC transporter involved in polysaccharide export. Proc Natl Acad Sci U S A 2007;104:19529-19534.

34. Cryz SJ, Mortimer PM, Mansfield V, Germanier R. Seroepidemiology of Klebsiella bacteremic isolates and implications for vaccine development. J Clin Microbiol 1986;23:687-690.

35. Tsay R-W, Siu LK, Fung C-P, Chang F-Y. Characteristics of bacteremia between communityacquired and nosocomial Klebsiella pneumoniae infection. Arch Intern Med 2002;162:10211027.

36. Jenney AW, Clements A, Farn JL, Wijburg OL, McGlinchey A, et al. Seroepidemiology of Klebsiella pneumoniae in an Australian tertiary hospital and its implications for vaccine development. J Clin Microbiol 2006;44:102-107.

37. Wyres KL, Nguyen TNT, Lam MMC, Judd LM, van Vinh Chau N, et al. Genomic surveillance for hypervirulence and multi-drug resistance in invasive Klebsiella pneumoniae from South and Southeast Asia. Genome Med 2020;12:11.

38. Lam MM., Wick R., Watts SC, Cerdeira LT, Wyres KL, et al. A genomic surveillance framework and genotyping tool for Klebsiella pneumoniae and its related species complex. Nat Commun 2021;12:4188.

39. Fang C, Shih Y, Cheong C, Yi W. Rapid and accurate determination of lipopolysaccharide Oantigen types in Klebsiella pneumoniae with a novel PCR-based O-genotyping method. 2015;54:666-675. 
40. Whitfield C, Richards JC, Perry MB, Clarke BR, Maclean LL. Expression of two structurally distinct D-galactan O-antigens in the lipopolysaccharide of Klebsiella pneumoniae serotype 01. J Bacteriol 1991;173:1420-1431.

41. Clarke BR, Whitfield C. Molecular cloning of the $\mathrm{rbb}$ region of Klebsiella pneumoniae serotype 01:K20: The rfb gene cluster is responsible for synthesis of the D-galactan I O polysaccharide. J Bacteriol 1992;174:4614-4621.

42. Sugiyama T, Kido N, Kato Y, Koide N, Yoshida T, et al. Evolutionary relationship among rfb gene clusters synthesizing mannose homopolymer as O-specific polysaccharides in Escherichia coli and Klebsiella pneumoniae. Gene 1997;198:111-113.

43. Kelly RF, Perry MB, MacLean LL, Whitfield C. Structures of the O-antigens of Klebsiella serotypes 02 (2a,2e), 02 (2a,2e,2h), and $02(2 \mathrm{a}, 2 \mathrm{f}, 2 \mathrm{~g})$, members of a family of related Dgalactan O-antigens in Klebsiella spp. J Endotoxin Res 1995;2:131-140.

44. Hsieh PF, Wu MC, Yang FL, Chen CT, Lou TC, et al. D-galactan II is an immunodominant antigen in 01 lipopolysaccharide and affects virulence in Klebsiella pneumoniae: Implication in vaccine design. Front Microbiol 2014;5:608.

45. Kelly SD, Clarke BR, Ovchinnikova OG, Sweeney RP, Williamson ML, et al. Klebsiella pneumoniae $\mathrm{O} 1$ and $\mathrm{O} 2 \mathrm{ac}$ antigens provide prototypes for an unusual strategy for polysaccharide antigen diversification. J Biol Chem 2019;294:10863-10876.

46. Clarke BR, Ovchinnikova OG, Kelly SD, Williamson ML, Jennifer E, et al. Molecular basis for the structural diversity in serogroup O2-antigen polysaccharides in Klebsiella pneumoniae. J Biol Chem 2018;293:4666-4679.

47. Bulati M, Busà R, Carcione C, lannolo G, Di Mento G, et al. Klebsiella pneumoniae lipopolysaccharides serotype $\mathrm{O}$ afg induce poor inflammatory immune responses ex vivo. Microorganisms 2021;9:1-15.

48. Thorpe H, Booton R, Kallonen T, Gibbon MJ, Couto N, et al. One Health or Three? Transmission modelling of Klebsiella isolates reveals ecological barriers to transmission between humans, animals and the environment. bioRxiv 2021;2021.08.05.455249.

49. Fostervold A, Hetland MAK, Bakksjø R, Bernhoff E, Holt KE, et al. A nationwide genomic study of clinical Klebsiella pneumoniae in Norway 2001-2015: Introduction and spread of ESBL facilitated by CG15 and CG307. bioRxiv 2021;2021.07.16.452602.

50. Raffelsberger N, Hetland MAK, Svendsen K, Småbrekke L, Löhr IH, et al. Gastrointestinal carriage of Klebsiella pneumoniae in a general adult population in Norway: a cross-sectional study of risk factors and bacterial genomic diversity. Gut Microbes 2021;13:1939599.

51. Leangapichart T, Lunha K, Jiwakanon J, Angkititrakul S, Järhult JD, et al. Characterization of Klebsiella pneumoniae complex isolates from pigs and humans in farms in Thailand: 
606
Population genomic structure, antibiotic resistance and virulence genes. J Antimicrob Chemother 2021;76:2012-2016.

52. Potter RF, Lainhart W, Twentyman J, Wallace MA, Wang B, et al. Population structure, antibiotic resistance, and Uropathogenicity of Klebsiella variicola. MBio 2018;9:e02481-18.

53. Wick RR, Schultz MB, Zobel J, Holt KE. Bandage: interactive visualization of de novo genome assemblies. Bioinformatics 2015;31:3350-3352.

54. Fu L, Niu B, Zhu Z, Wu S, Li W. CD-HIT: Accelerated for clustering the next-generation sequencing data. Bioinformatics 2012;28:3150-3152.

55. Seemann T. Prokka: rapid prokaryotic genome annotation. Bioinformatics 2014;30:20682069.

56. Carver TJ, Rutherford KM, Berriman M, MA R, Barrell BG, et al. ACT: the Artemis comparison tool. Bioinformatics 2005;21:3422-3423.

57. Siguier P, Perochon J, Lestrade L, Mahillon J, Chandler M. ISfinder: the reference centre for bacterial insertion sequences. Nucleic Acids Res 2006;34:D32-D36.

58. Gilchrist CLM, Chooi Y-H. clinker \& clustermap.js: automatic generation of gene cluster comparison figures. Bioinformatics 2021; Epub ahead of print.

59. Rahn A, Beis K, Naismith JH, Whitfield C. A novel outer membrane protein, Wzi, is involved in surface assembly of the Escherichia coli K30 group 1 capsule. J Bacteriol 2003;185:5882-90.

60. Sathiyamoorthy K, Mills E, Franzmann TM, Rosenshine I, Saper MA. The crystal structure of Escherichia coli group 4 capsule protein $\mathrm{GFCC}$ reveals a domain organization resembling that of Wza. Biochemistry 2011;50:5465-5476.

61. Ferrières L, Aslam SN, Cooper RM, Clarke DJ. The yjbEFGH locus in Escherichia coli K-12 is an operon encoding proteins involved in exopolysaccharide production. Microbiology 2007;153:1070-1080.

62. Holt KE, Lassalle F, Wyres KL, Wick R, Mostowy RJ. Diversity and evolution of surface polysaccharide synthesis loci in Enterobacteriales. ISME J 2020;14:1713-1730.

63. Hansen DS, Mestre F, Dome A, Toma JM. Klebsiella pneumoniae lipopolysaccharide O typing: Revision of prototype strains and O-group distribution among clinical isolates from different sources and countries. J Clin Microbiol 1999;37:56-62.

64. Choi M, Hegerle N, Nkeze J, Sen S, Jamindar S, et al. The diversity of lipopolysaccharide (O) and capsular polysaccharide (K) antigens of invasive Klebsiella pneumoniae in a multi-country collection. Front Microbiol 2020;11:1249. 